\title{
Bufalin induces DNA damage response under hypoxic condition in myeloma cells
}

\author{
ERI FUJII $^{1,2}$, YUKI INADA ${ }^{1}$, MISAKI KAKOKI ${ }^{1}$, NAO NISHIMURA ${ }^{3}$, SHINYA ENDO $^{3}$, SHIHO FUJIWARA $^{3}$, \\ NAOKO WADA $^{3}$, YAWARA KAWANO ${ }^{3}$, YUTAKA OKUNO ${ }^{3}$, TOSHIYA SUGIMOTO ${ }^{1}$ and HIROYUKI HATA ${ }^{3,4}$ \\ ${ }^{1}$ Graduate School of Health Sciences, Course of Medical Laboratory Sciences, Kumamoto University, \\ Kumamoto 862-0976; ${ }^{2}$ Department of Clinical Laboratory, Osaka University Hospital, Suita, Osaka 565-0871; \\ ${ }^{3}$ Department of Hematology, Faculty of Medical Sciences, Kumamoto University, Kumamoto 860-8556; \\ ${ }^{4}$ Division of Informative Clinical Sciences, Faculty of Medical Sciences, Kumamoto University, Kumamoto 862-0976, Japan
}

Received September 18, 2017; Accepted February 13, 2018

DOI: $10.3892 /$ ol.2018.8091

\begin{abstract}
Hypoxia serves a crucial role in the development of drug resistance in various cancer cells. Therefore, many attempts targeting hypoxia are underway to overcome the drug resistance mediated by hypoxia. This strategy is useful for multiple myeloma (MM) cells, as MM cells reside within the bone marrow, where oxygen concentrations are relatively low. A natural compound library was screened to identify compounds exerting cytotoxicity in MM cells under hypoxic conditions. Bufalin exhibited marked cytotoxicity to MM cells under normoxic and hypoxic conditions. No significant toxicity was observed in lymphocytes obtained from healthy donors. Under normoxic conditions, bufalin induced a DNA double strand break (DSB) response, ROS induction and apoptosis within 24 with a rapid response compared with melphalan. Interestingly, the bufalin-induced DSB response was not impaired by low oxygen concentrations while the DSB response by melphalan was reduced. Furthermore, treatment with bufalin abolished HIF-1 $\alpha$ expression under hypoxia, suggesting that bufalin exerts cytotoxicity under hypoxia by regulating HIF-1 $\alpha$. These results indicate that bufalin induces apoptosis in MM cells through DSB under hypoxic conditions by inhibiting HIF-1 $\alpha$, suggesting that bufalin could be useful for eradication of drug-resistant MM cells in the hypoxic microenvironment.
\end{abstract}

\section{Introduction}

Multiple myeloma (MM) is a hematological tumor of plasma cells that shows characteristic expansion of tumor cells within

Correspondence to: Professor Hiroyuki Hata, Division of Informative Clinical Sciences, Faculty of Medical Sciences, Kumamoto University, Kuhonji 4-24-1, Chuoku, Kumamoto City, Kumamoto 862-0976, Japan

E-mail: hata@kumamoto-u.ac.jp

Key words: multiple myeloma, bufalin, DNA damage, apoptosis, HIF-1 $\alpha$, hypoxia the bone marrow. Since the microenvironment within bone marrow is unique in terms of cytokine production and its interaction with various cells including MM cells, these environments can influence the survival and drug resistance of MM cells (1-3). In particular, oxygen concentration in the microenvironment is one of the greatest factors controlling survival of MM cells (4).

Oxygen plays an important role for MM cells by influencing the sensitivity to chemotherapeutic reagents. Previous studies showed that the sensitivity of MM cells to melphalan is reduced by hypoxia (5). Similar findings with other chemotherapeutic drugs have also been reported in various solid tumors (6-9). Therefore, these data suggest the existence of universal mechanisms by which oxygen concentration can regulate drug sensitivity. Notably, it is well known that oxygen concentration is extremely low in bone marrow, where MM cells survive and grow, and that drug-resistant MM cells can be generated within bone marrow, thus contributing to recurrence of the disease. Therefore, manipulation of hypoxia-induced drug resistance should be essential to improve survival of MM patients.

In this study, we searched for chemical compounds with the ability to kill myeloma cells under hypoxic conditions. We performed drug screening using a natural compound library and identified bufalin, a component of a Chinese medicine (Chan Su). We also analyzed the molecular mechanisms underlying the cytotoxicity of bufalin to MM cells under hypoxic conditions which has not been reported.

\section{Materials and methods}

Screening of compounds. A natural compound library consisting of 258 compounds was obtained from the Institute of Natural Medicine, Toyama University (Toyama, Japan). Each compound was supplemented to myeloma cell lines at a concentration of $10 \mu \mathrm{M}$ and treated cells were cultured for 5 days under $1 \%$ oxygen concentration using a hypoxic culture chamber (Astec, Fukuoka, Japan).

Cell lines and cell culture. Two human myeloma cell lines, KMS12PE (10) and U266 (11) were utilized. Cells were cultured in RPMI-1640 medium supplemented with $10 \%$ fetal calf serum at $37^{\circ} \mathrm{C}$ with humidified air. Human peripheral 
A

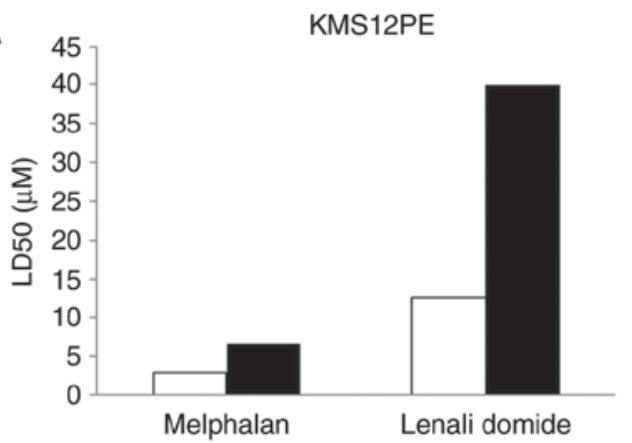

B

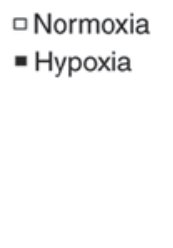

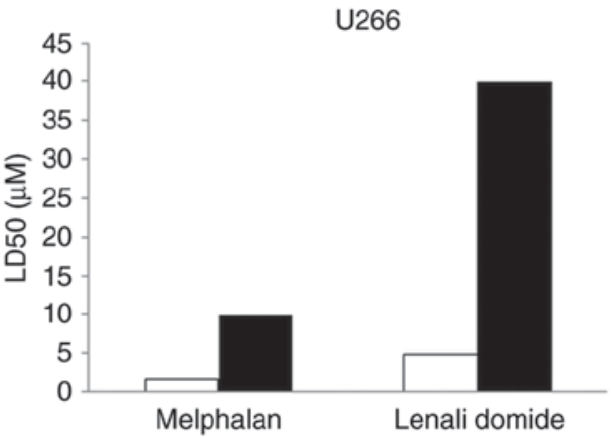

口Normoxia

- Hypoxia

Figure 1. Induction of drug resistance for melphalan and lenalidomide under hypoxia conditions. (A) KMS12PE cells and (B) U266 cells were incubated with melphalan or lenalidomide for $72 \mathrm{~h}$ under normoxic or hypoxic conditions. Cytotoxicity was quantified by WST8 assay and LD50 was calculated. Open bars: Normoxia. Solid bars: Hypoxia. A significant increase of LD50 was observed by exposure of cells to hypoxia.

blood mononuclear cells (PMBCs) were obtained from a healthy volunteer and purified by Ficoll density gradation.

Cytotoxic and apoptosis assays. Cytotoxicity of the compounds was analyzed by Cell Counting Kit- 8 (Dohjin Chemicals, Kumamoto, Japan) or trypan blue dye exclusion assay according to the manufacturer's protocol. The MEBCYTO Apoptosis kit was used to detect apoptosis by flow cytometry using FACSVerse (BD Biosciences, Franklin Lakes, NJ, USA) according to the manufacturer's protocol. Melphalan and lenalidomide were purchased from Santa Cruz Biotechnology, Inc. (Santa Cruz, CA, USA) and Sigma-Aldrich; Merck KGaA, (Darmstadt, Germany), respectively. A pan-caspase inhibitor, ZVAD-fmk (Medical and Biological Laboratories, Nagoya, Japan), was utilized in some experiment at a concentration of $32 \mathrm{nM}$.

Detection of reactive oxygen species (ROS). Production of ROS was analyzed using CellROX flow cytometry assay kits (Thermo Fisher Scientific, Yokohama, JAPAN) and FACS Verse according to the manufacturer's protocols.

Western blotting. Cell lysates were obtained by lysing cells with M-PER mammalian protein extraction reagent (Thermo Fisher Scientific, Inc., Yokohama, Japan) supplemented with phosphatase inhibitor cocktail and protease inhibitor cocktail (Nacalai Tesque, Kyoto, Japan). Antibodies for PARP, $\gamma$-H2A.X and HIF-1 $\alpha$ were purchased from Cell Signaling Technology, Inc. (Tokyo, Japan).

Statistical analysis. Statistical analysis between two groups was performed by Student's t-test. For multiple group comparisons, one-way or two-way ANOVA was performed. The data were analyzed statistically by using GraphPad Prism software (GraphPad Software, Inc., La Jolla, CA, USA) and P<0.05 was considered to indicate a statistically significant difference.

\section{Results}

To confirm that myeloma cells show drug resistance under hypoxic conditions, we treated myeloma cells with melphalan and lenalidomide under normoxic or hypoxic conditions (Fig. 1). The LD50 values of melphalan in both KMS12PE and U266 cells under normoxic conditions were 3.0 and $1.8 \mu \mathrm{M}$ and those of lenalidomide were 12.6 and $4.9 \mu \mathrm{M}$,

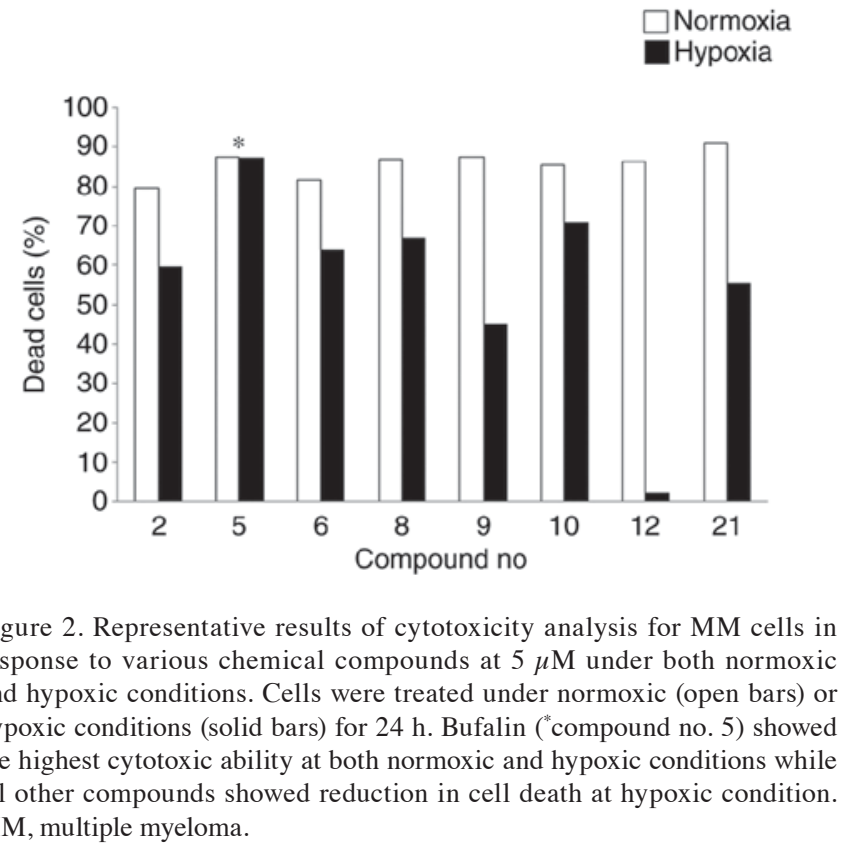

respectively. Notably, the LD50 values of these reagents were elevated at hypoxic conditions. The LD50 values of melphalan in KMS12PE and U266 cells under hypoxic conditions were 6.7 and $9.9 \mu \mathrm{M}$, respectively, and the LD50 values of lenalidomide in KMS12PE and U266 cells were above $40 \mu \mathrm{M}$. Together these data indicate that myeloma cells showed drug resistance for these two reagents under low concentrations of oxygen.

We next screened 258 compounds in a natural compound library under hypoxic conditions in KMS12PE and U266 cells and found that 8 compounds induced significant cell death of more than $50 \%$ of the whole cell population. We analyzed cytotoxicity of each of these compounds in KMS12PE cells under both normoxic and hypoxic conditions and found that bufalin exerted high cytotoxicity at both conditions (compound no. 5 in Fig. 2) while other compounds showed reduction in cell death at hypoxic condition.

We thus further analyzed bufalin as a potential candidate that retains cytotoxicity regardless of oxygen concentration. A myeloma cell line, KMS12PE, was treated by bufalin at various concentrations either under normoxic or hypoxic 

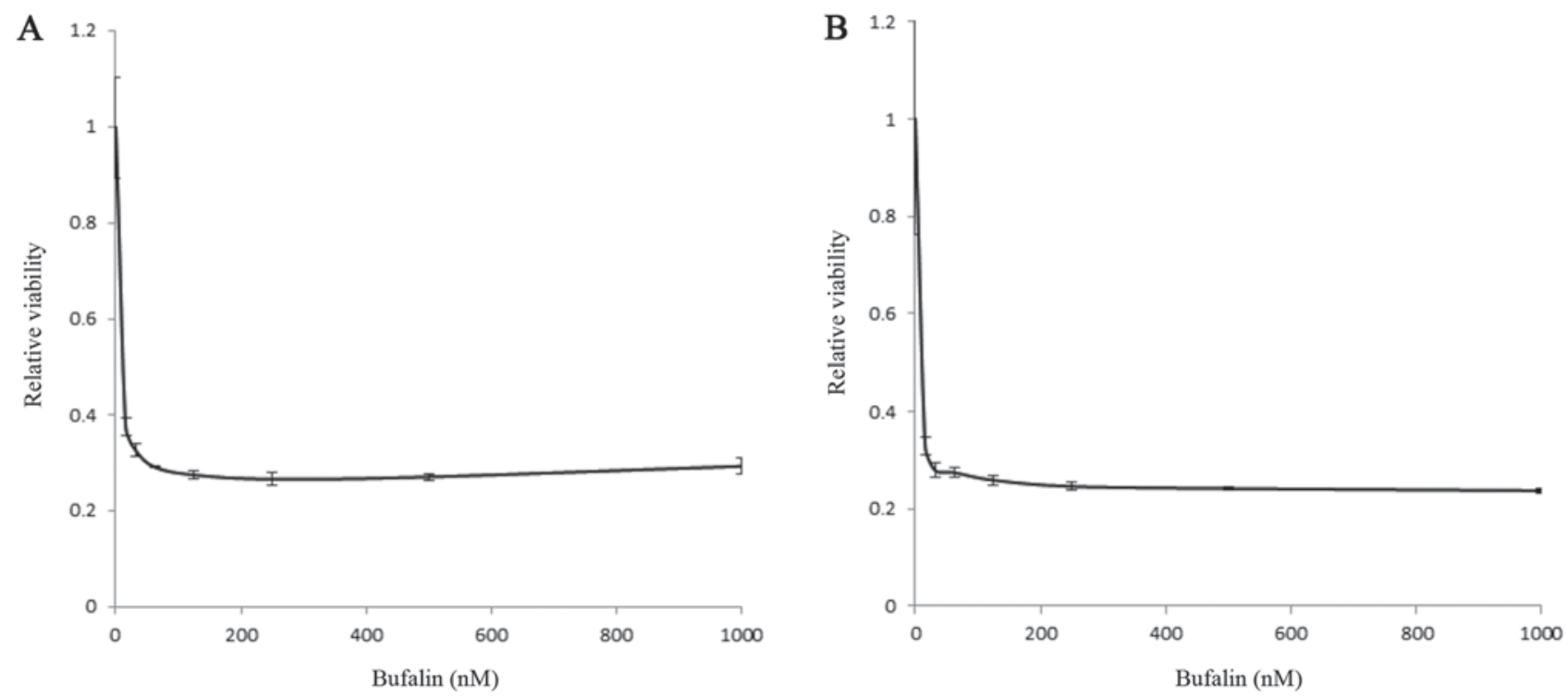

Figure 3. Cytotoxic effect of bufalin in MM cells either at (A) normoxic or (B) hypoxic conditions. KMS12PE cells were incubated with various concentrations of bufalin for $24 \mathrm{~h}$. Cytotoxicity was evaluated by using cell counting kit (solid column) at 31.25, 62.5, 125, 250, 500 and 1,000 nM of bufalin. Bufalin induced cytotoxicity at dose dependent manner either at normoxic and hypoxic conditions. There were no statistical differences in cytotoxicity regardless oxygen concentration ( $\mathrm{P}=0.9757$, analyzed by two-way ANOVA). MM, multiple myeloma.

conditions. As shown in Fig. 3, bufalin induced significant cytotoxicity below micro-molar levels independent of oxygen concentrations.

To elucidate whether bufalin shows toxicity to freshly isolated cells, MM cells obtained from patient bone marrow and PBMCs obtained from healthy volunteer were cultured and treated with bufalin. The results showed that bufalin induced significant cell death to primary MM cells, while it did not induce cell death in PBMCs as much as those observed in primary MM cells $(\mathrm{P}<0.0001$, analyzed by two-way Anova) (Fig. 4). Together this suggests that bufalin may not show toxicity to non-malignant cells.

To further characterize the cytotoxicity by bufalin, we performed Annexin V/PI analysis of KMS12PE cells treated with $32 \mathrm{nM}$ bufalin for $24 \mathrm{~h}$ either at normoxic or hypoxic conditions. Bufalin induced both early and late phase apoptosis and this increase in apoptosis was reduced by treatment with a caspase inhibitor (Fig. 5). These results indicate that the cytotoxicity induced by bufalin involves apoptosis mediated by caspase activation either in normoxic and hypoxic conditions.

As ROS is an inducer of apoptosis, we next analyzed the production of ROS in response to bufalin treatment. We observed a significant induction of ROS with bufalin treatment, and this induction was partially inhibited by $\mathrm{N}$-acetyl cysteine (Fig. 6). This finding indicates that ROS production may be one of the contributors for the cytotoxic effects of bufalin.

Since several reports previously showed that bufalin induces DNA double strand breaks (DSBs) (12-14), we analyzed $\gamma-\mathrm{H} 2 \mathrm{AX}$ induction, which is a hallmark of the DSB response. As shown in Fig. 7A, bufalin induced $\gamma$-H2AX in myeloma cells in a time-dependent manner under normoxic conditions. We also examined DSB induction under hypoxia conditions, and found induction of $\gamma-\mathrm{H} 2 \mathrm{AX}$ by bufalin as found at normoxia (Fig. 7B). However, induction of $\gamma$-H2AX by melphalan at normoxia was found at fewer amounts and delayed as compared to bufalin. Moreover, melphalan-induced $\gamma-\mathrm{H} 2 \mathrm{AX}$ even impaired at hypoxic conditions compared with normoxic conditions. Because melphalan did not show anti-tumor effect not earlier than $24 \mathrm{~h}$, western blot analysis at earlier occasions is not shown. Cleavage of PARP was also observed in response to bufalin treatment regardless of oxygen concentration, while melphalan-inducing PARP cleavage was inhibited at hypoxic conditions (Fig. 7A and B).

Since HIF- $1 \alpha$ is induced and considered to contribute drug resistance in hypoxia, we evaluated whether bufalin modulates HIF-1 $\alpha$ under hypoxic conditions. Interestingly, treatment of myeloma cells with bufalin at $16 \mathrm{nM}$ for $24 \mathrm{~h}$ significantly inhibited the expression of HIF-1 $\alpha$ in hypoxic conditions (Fig. 8), suggesting that the cytotoxic effect of bufalin under hypoxia may involve the reduction of HIF-1 $\alpha$.

\section{Discussion}

Oxygen concentration plays a crucial role in the survival of cancer cells. Low oxygen concentration induces expression of numerous genes regulated by HIF- $1 \alpha$, a master transcriptional factor under hypoxic conditions. One of the features mediated by hypoxia is acquisition of drug resistance. Previous reports demonstrated acquisition of drug resistance by hypoxia in various types of cancer cells $(4,7-9,15)$. Mechanisms regulating drug resistance under hypoxia have been described, involving microRNAs (16), the multi-drug resistant (MDR) gene $(15,17)$ or the autophagy pathway (18). Modulating these pathways is thought to re-sensitize tumor cells to anti-cancer reagents.

Previous studies have examined the correlation between hypoxia and cancer stem cells other than drug resistance (19). Hypoxic conditions can transform tumor cells to cancer stem cells and are required for the maintenance of cancer stem cells (20-22). Targeting cancer stem cells should be important to eradicate tumor cells. We previously 


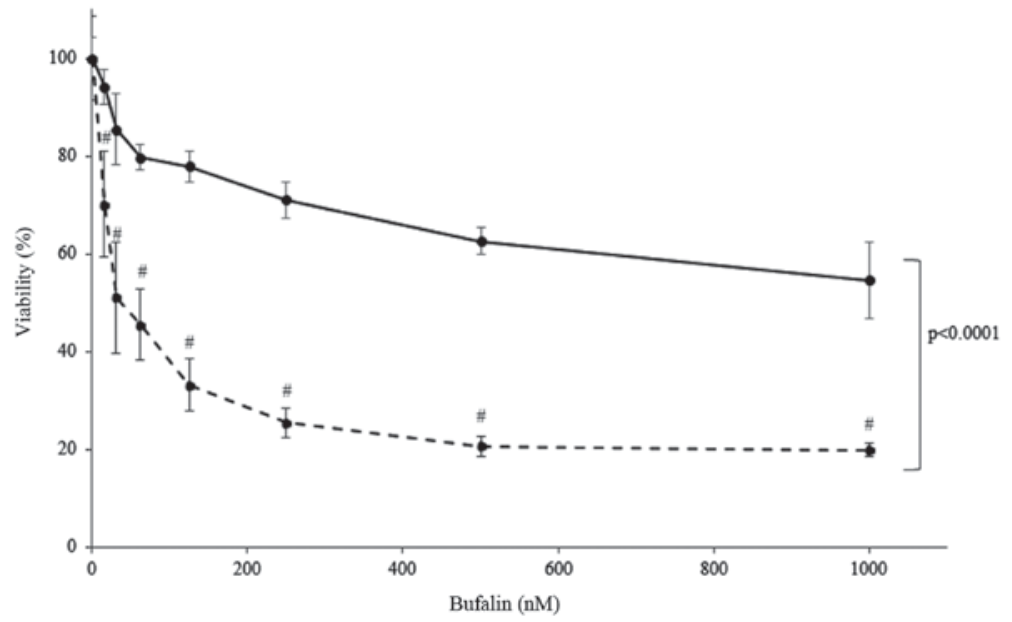

Figure 4. Cytotoxic effect of bufalin in primary myeloma cells (dotted line) and PBMCs from a healthy donor (solid line) under normoxic conditions. Cells were incubated with the indicated concentration of bufalin at $15.625,31.25,62.5,125,250,500$ and $1,000 \mathrm{nM}$ for $24 \mathrm{~h}$ and cytotoxicity was evaluated by cell counting kit. Freshly isolated myeloma cells showed marked cytotoxicity by bufalin at dose dependent manner $\left({ }^{*} \mathrm{P}<0.0001\right.$ compared to bufalin $0 \mathrm{nM}$, analyzed by one-way ANOVA and Bonferroni multiple comparison test) while viability of PBMCs was retained more than $60 \%$, suggesting PBMC is less susceptible to bufalin than MM cells ( $\mathrm{P}<0.0001$, analyzed by two-way ANOVA and Bonferroni multiple comparison test). PBMC, peripheral blood mononuclear cell; MM, multiple myeloma.
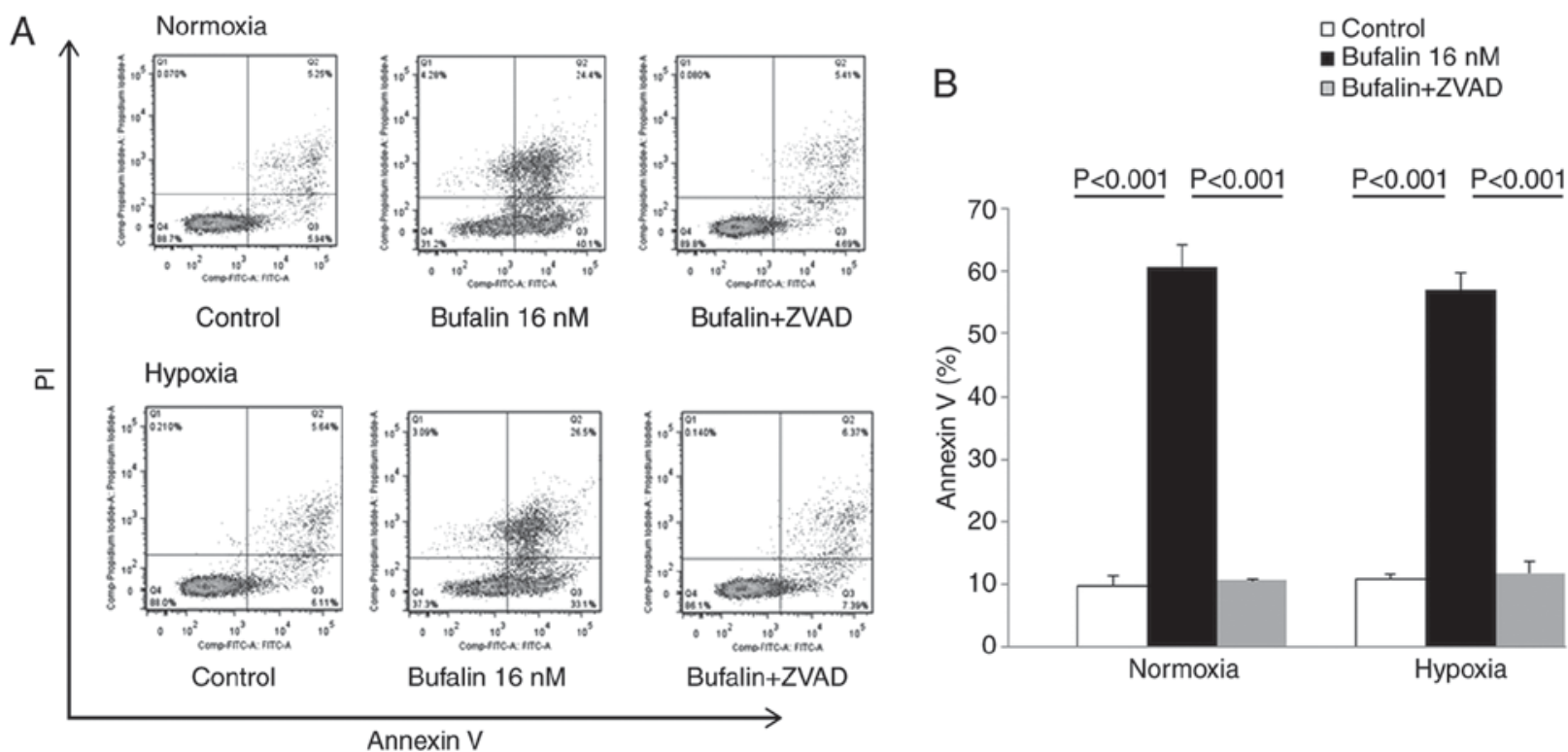

Figure 5. Induction of apoptosis in KMS12PE cells treated with bufalin either under normoxic or hypoxic conditions. KMS12PE cells were treated with bufalin alone or in combination with a pan-caspase inhibitor (ZVAD) for $24 \mathrm{~h}$ under normoxic or hypoxic conditions and apoptosis was evaluated by Annexin V/PI staining. (A) Representative results from flow cytometry analyses. (B) Graphical presentation of the Annexin V-positive population calculated from flow cytometry results in (A). Apoptosis, induced by bufalin, was inhibited by caspase-inhibitor at both normoxic and hypoxic conditions $(\mathrm{P}<0.001$, analyzed by one-way ANOVA and Bonferroni multiple comparison test).

reported that hypoxic conditions transform myeloma cells to an immature phenotype, suggesting that myeloma stem cells may adapt to hypoxic conditions (23). Because melphalan and lenalidomide showed reduced efficacy at hypoxic conditions as shown in this report, our results suggest that these treatments may not retain the ability to target myeloma stem cells, although we did not perform confirmatory experiments using myeloma stem cells this time. Recently, Sun et al, reported that bufalin inhibits stemness and overcome drug resistance of colon cancer cells (24). This finding enforces our results and warrants usage of bufalin to exterminate myeloma stem cells.
We found, for the first time, that bufalin induced the DSB response in MM cells independent of oxygen concentrations. Although DSB response mediated by bufalin at normoxic condition has scarcely been reported in solid tumors, especially interacting to topoisomerase II (12-14), the function of bufalin under hypoxic conditions has not been fully elucidated. Only two previous reports described a correlation between bufalin and hypoxia in colon, cervical and hepatocellular carcinoma cells, and these studies suggested that reduction of HIF-1 could be involved in the cytotoxicity induced by bufalin $(25,26)$. Interestingly, we found a decrease of HIF-1 $\alpha$ in response to treatment with bufalin under hypoxic conditions in MM cells. 

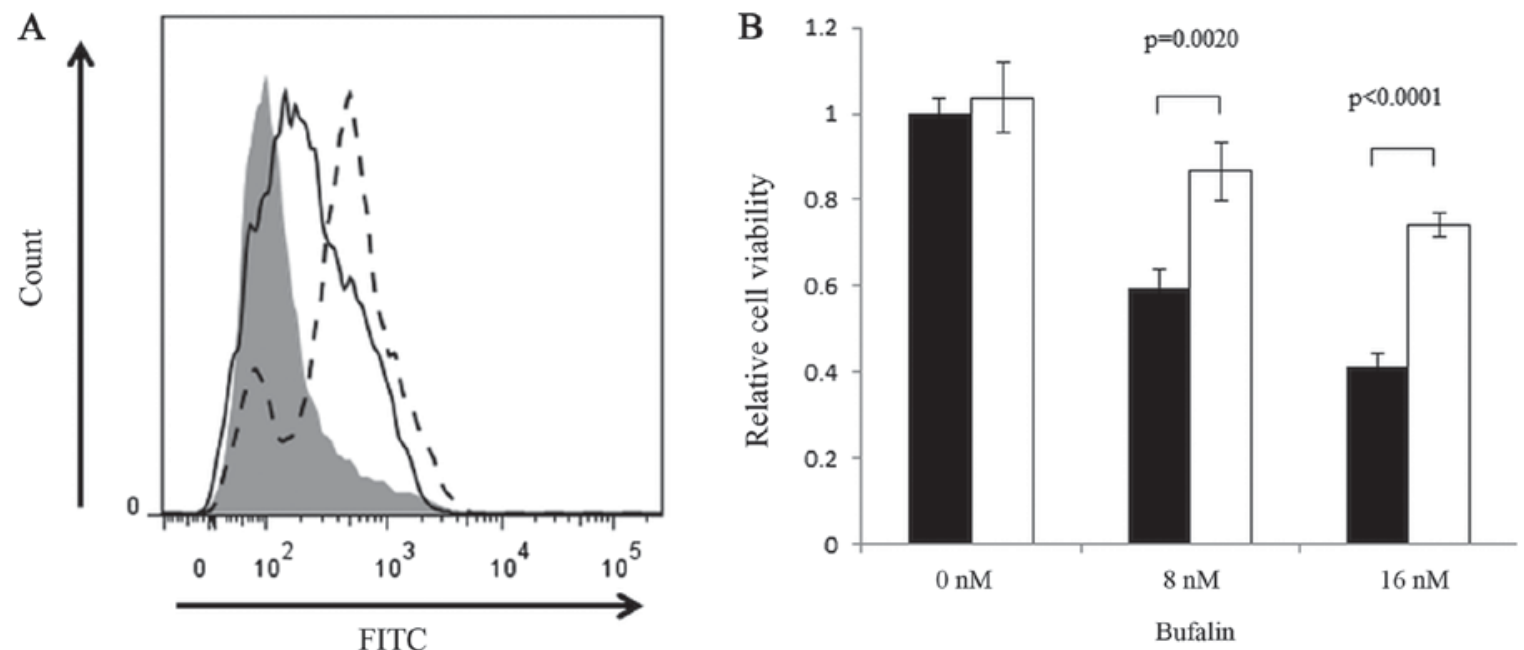

Figure 6. Induction of ROS by bufalin. (A) ROS was evaluated by using CellROX ${ }^{\circledast}$ Flow Cytometry Assay kits and flowcytometry. Shaded area, Negative control; Dotted line, Positive control (tert-butyl hydroperoxide: TBHP); Solid line, Bufalin. (B) Cytotoxicity by bufalin was hampered by the treatment with $\mathrm{N}$-Acetyl Cystein (NAC at $5 \mathrm{mM}$ ). Cell viability was analyzed by cell counting kit. Shaded bars, Bufalin; white bars, Bufalin+NAC. Bufailn induced significant cell death $(\mathrm{P}<0.01)$ at 8 and $16 \mathrm{nM}$ while this was partially reduced by NAC. ROS, reactive oxygen species.

A

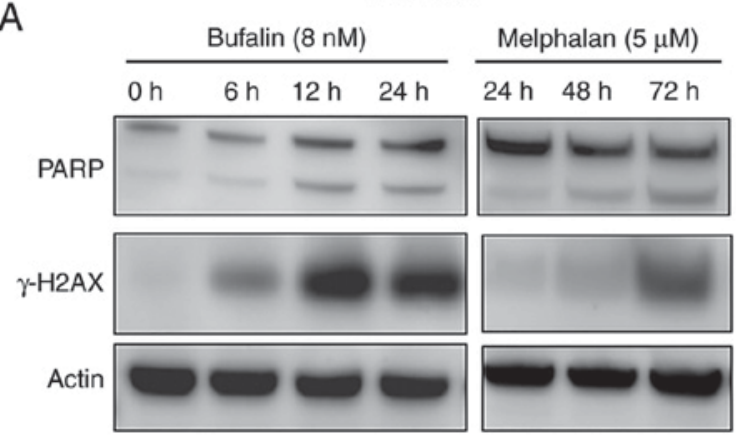

B

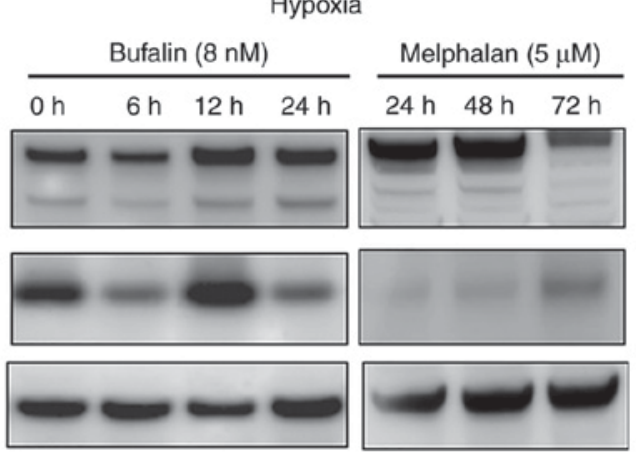

Figure 7. Induction of PARP cleavage and $\gamma$-H2AX by bufalin or melphalan. Western blot analysis of KMS12PE cells treated with either bufalin ( 8 nM) or melphalan $(5 \mu \mathrm{M})$ for indicated periods of time under (A) normoxia or (B) hypoxia. At normoxic condition, PARP was cleaved by the treatment with bufalin as early as $6 \mathrm{~h}$ while it was observed at $72 \mathrm{~h}$ when treated by melphalan. Earlier induction of $\gamma$-H2AX was observed according to cleavage of PARP at normoxic condition by bufalin compared to melphalan (A). These phenomena were not affected at hypoxic condition in bufalin-treated cells while these were reduced in melphalan-treated cells (B).

As a growing body of evidence has shown a central role of hypoxia in the acquisition of drug resistance, targeting HIF-1 $\alpha$ is now considered to be an important approach to overcome drug resistance $(27,28)$. Indeed, targeting HIF-1 $\alpha$ sensitizes myeloma cells to chemotherapeutic agents and reduces the number of myeloma stem cells $(5,29)$. Our finding of HIF-1 $\alpha$ inhibition by bufalin should contribute to the development of a HIF-1 $\alpha$ targeted strategy for treatment of MM. The demonstrated cytotoxicity by bufalin regardless of oxygen concentration enforces the significance of bufalin in the treatment of cancers survive within hypoxic niche other than myeloma. However, we did not evaluated ubiquitination or degradation of HIF-1 by the treatment with bufalin at this time. Regulatory effect of bufalin in expression of HIF-1 remains to be elucidated.

Adverse events, especially cardiotoxicity (30), caused by bufalin is an important issue when using this reagent at clinical setting. However, the concentration inducing cardiotoxicity was estimated as $1 \mathrm{mM}$ which was significantly higher than those exerting anti-tumor effect presented in this manuscript,

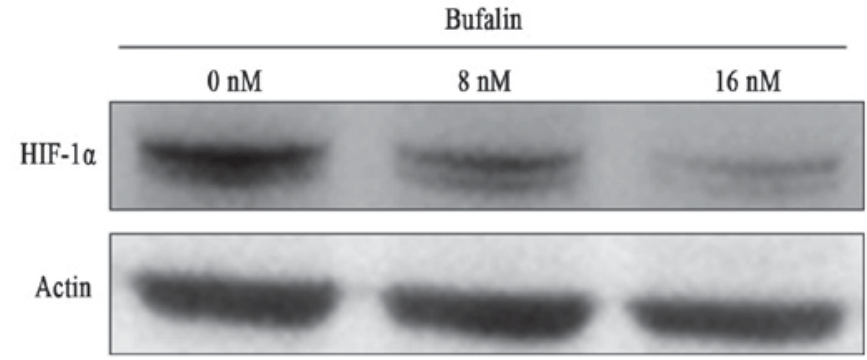

Figure 8. Bufalin inhibited expression of HIF-1 $\alpha$ under hypoxia. KMS12PE cells were treated with or without bufalin under hypoxic conditions for $24 \mathrm{~h}$. HIF-1 $\alpha$ expression was analyzed by western blot analysis.

suggesting clinical usage of bufalin might spare cardiotoxicity. Indeed, there have been several reports showing in vivo findings $(31,32)$. Given the low working concentration of bufalin, as low as nano-molar levels, the achievement of an effective concentration of bufalin should be possible. Successful in vivo treatment of an osteosarcoma-bearing mouse by bufalin 
suggests its future usage in the clinical setting (33). In addition, our findings demonstrating the low toxicity of bufalin in normal PBMCs implies a low toxicity profile for normal tissues.

\section{Acknowledgements}

The authors would like to thank Dr Gabrielle White Wolf for editing a draft of this manuscript.

\section{Funding}

This study was supported by in part by a grant from the Japanese Amyloidosis Research Committee from the Ministry of Health, Labor and Welfare (grant no. ippan-022).

\section{Availability of data and materials}

The datasets used and/or analyzed during the current study are available from the corresponding author on reasonable request.

\section{Authors' contributions}

EF, YI and MK executed the experiments. NN, SE, SF, NW, YO and TS supervised the experimental procedures and analysed the results. YK performed the statistical analysis. $\mathrm{HH}$ conducted experiments, organized the discussions and finalized the manuscript.

\section{Ethics approval and consent to participate}

Not applicable.

\section{Consent for publication}

Not applicable.

\section{Competing interests}

The authors declare that they have no competing interests.

\section{References}

1. Di Marzo L, Desantis V, Solimando AG, Ruggieri S, Annese T, Nico B, Fumarulo R, Vacca A and Frassanito MA: Microenvironment drug resistance in multiple myeloma: Emerging new players. Oncotarget 7: 60698-60711, 2016.

2. Kawano Y, Moschetta M, Manier S, Glavey S, Görgün GT, Roccaro AM, Anderson KC and Ghobrial IM: Targeting the bone marrow microenvironment in multiple myeloma. Immunol Rev 263: 160-172, 2015.

3. Wang J, Faict S, Maes K, De Bruyne E, Van Valckenborgh E, Schots R, Vanderkerken K and Menu E: Extracellular vesicle cross-talk in the bone marrow microenvironment: Implications in multiple myeloma. Oncotarget 7: 38927-38945, 2016.

4. Garvalov BK and Acker T: Implications of oxygen homeostasis for tumor biology and treatment. Adv Exp Med Biol 903: 169-185, 2016.

5. Hu Y, Kirito K, Yoshida K, Mitsumori T, Nakajima K, Nozaki Y, Hamanaka S, Nagashima T, Kunitama M, Sakoe K and Komatsu N: Inhibition of hypoxia-inducible factor-1 function enhances the sensitivity of multiple myeloma cells to melphalan. Mol Cancer Ther 8: 2329-2338, 2009.

6. Daster S, Amatruda N, Calabrese D, Ivanek R, Turrini E, Droeser RA, Zajac P, Fimognari C, Spagnoli GC, Iezzi G, et al: Induction of hypoxia and necrosis in multicellular tumor spheroids is associated with resistance to chemotherapy treatment. Oncotarget 8: 1725-1736, 2017
7. Zhao W, Xia SQ, Zhuang JP, Zhang ZP, You CC, Yan JL and $\mathrm{Xu}$ GP: Hypoxia-induced resistance to cisplatin-mediated apoptosis in osteosarcoma cells is reversed by gambogic acid independently of HIF-1 $\alpha$. Mol Cell Biochem 420: 1-8, 2016.

8. Qin Y, Roszik J, Chattopadhyay C, Hashimoto Y, Liu C, Cooper ZA, Wargo JA, Hwu P, Ekmekcioglu S and Grimm EA: Hypoxia-driven mechanism of vemurafenib resistance in melanoma. Mol Cancer Ther 15: 2442-2454, 2016.

9. Mao XG, Wang C, Liu DY, Zhang X, Wang L, Yan M, Zhang W, Zhu J, Li ZC, Mi C, et al: Hypoxia upregulates HIG2 expression and contributes to bevacizumab resistance in glioblastoma. Oncotarget 7: 47808-47820, 2016.

10. Ohtsuki T, Yawata Y, Wada H, Sugihara T, Mori M and Namba M: Two human myeloma cell lines, amylase-producing KMS-12-PE and amylase-non-producing KMS-12-BM, were established from a patient, having the same chromosome marker, $\mathrm{t}(11 ; 14)(\mathrm{q} 13 ; \mathrm{q} 32)$. Br J Haematol 73: 199-204, 1989.

11. Ikeyama S, Nakagawa S, Arakawa M, Sugino $\mathrm{H}$ and Kakinuma A: Purification and characterization of IgE produced by human myeloma cell line, U266. Mol Immunol 23: 159-167, 1986.

12. Pastor $\mathrm{N}$ and Cortés $\mathrm{F}$ : Bufalin influences the repair of X-ray-induced DNA breaks in Chinese hamster cells. DNA Repair (Amst) 2: 1353-1360, 2003.

13. Wu SH, Hsiao YT, Chen JC, Lin JH, Hsu SC, Hsia TC, Yang ST, Hsu WH and Chung JG: Bufalin alters gene expressions associated DNA damage, cell cycle and apoptosis in human lung cancer NCI-H460 cells in vitro. Molecules 19: 6047-6057, 2014.

14. Wu SH, Wu TY, Hsiao YT, Lin JH, Hsu SC, Hsia TC, Yang ST, Hsu WH and Chung JG: Bufalin induces cell death in human lung cancer cells through disruption of DNA damage response pathways. Am J Chin Med 42: 729-742, 2014.

15. Li D, Zhou L, Huang J and Xiao X: Effect of multidrug resistance $1 / \mathrm{P}$-glycoprotein on the hypoxia-induced multidrug resistance of human laryngeal cancer cells. Oncol Lett 12: 1569-1574, 2016.

16. Zhou C, Tan W, Lv H, Gao F and Sun J: Hypoxia-inducible microRNA-488 regulates apoptosis by targeting Bim in osteosarcoma. Cell Oncol (Dordr) 39: 463-471, 2016.

17. Chen YL, Yang TY, Chen KC, Wu CL, Hsu SL and Hsueh CM: Hypoxia can impair doxorubicin resistance of non-small cell lung cancer cells by inhibiting MRP1 and P-gp expression and boosting the chemosensitizing effects of MRP1 and P-gp blockers. Cell Oncol (Dordr) 39: 411-433, 2016

18. Feng H, Wang J, Chen W, Shan B, Guo Y, Xu J, Wang L, Guo P and Zhang Y: Hypoxia-induced autophagy as an additional mechanism in human osteosarcoma radioresistance. J Bone Oncol 5: 67-73, 2016.

19. Mohyeldin A, Garzón-Muvdi T and Quiñones-Hinojosa A: Oxygen in stem cell biology: A critical component of the stem cell niche. Cell Stem Cell 7: 150-161, 2010.

20. Hoshino H, Nagano H, Haraguchi N, Nishikawa S, Tomokuni A, Kano Y, Fukusumi T, Saito T, Ozaki M, Sakai D, et al: Hypoxia and TP53 deficiency for induced pluripotent stem cell-like properties in gastrointestinal cancer. Int J Oncol 40: 1423-1430, 2012.

21. Li Z and Rich JN: Hypoxia and hypoxia inducible factors in cancer stem cell maintenance. Curr Top Microbiol Immunol 345: 21-30, 2010

22. Ma Y, Liang D, Liu J, Axcrona K, Kvalheim G, Stokke T, Nesland JM and Suo Z: Prostate cancer cell lines under hypoxia exhibit greater stem-like properties. PLoS One 6: e29170, 2011.

23. Kawano Y, Kikukawa Y, Fujiwara S, Wada N, Okuno Y, Mitsuya $\mathrm{H}$ and Hata $\mathrm{H}$ : Hypoxia reduces $\mathrm{CD} 138$ expression and induces an immature and stem cell-like transcriptional program in myeloma cells. Int J Oncol 43: 1809-1816, 2013.

24. Sun J, Xu K, Qiu Y, Gao H, Xu J, Tang Q and Yin P: Bufalin reverses acquired drug resistance by inhibiting stemness in colorectal cancer cells. Oncol Rep 38: 1420-1430, 2017.

25. Wang H, Zhang C, Xu L, Zang K, Ning Z, Jiang F, Chi H, Zhu X and Meng Z: Bufalin suppresses hepatocellular carcinoma invasion and metastasis by targeting HIF-1 $\alpha$ via the PI3K/AKT/mTOR pathway. Oncotarget 7: 20193-20208, 2016.

26. Xie CM, Liu XY, Yu S and Cheng CH: Cardiac glycosides block cancer growth through HIF-1 $\alpha$ - and NF- $\kappa \mathrm{B}$-mediated Plk1. Carcinogenesis 34: 1870-1880, 2013.

27. Jahanban-Esfahlan R, de la Guardia M, Ahmadi D and Yousefi B: Modulating tumor hypoxia by nanomedicine for effective cancer therapy. J Cell Physiol 233: 2019-2031, 2017.

28. Yu T, Tang B and Sun X: Development of inhibitors targeting hypoxia-inducible factor 1 and 2 for cancer therapy. Yonsei Med J 58: 489-496, 2017. 
29. Borsi E, Terragna C, Brioli A, Tacchetti P, Martello M and Cavo M: Therapeutic targeting of hypoxia and hypoxia-inducible factor 1 alpha in multiple myeloma. Transl Res 165: 641-650, 2015.

30. Bick RJ, Poindexter BJ, Sweney RR and Dasgupta A: Effects of Chan $\mathrm{Su}$, a traditional Chinese medicine, on the calcium transients of isolated cardiomyocytes: Cardiotoxicity due to more than Na, K-ATPase blocking. Life Sci 72: 699-709, 2002.

31. Liu M, Feng LX, Sun P, Liu W, Wu WY, Jiang BH, Yang M, Hu LH, Guo DA and Liu X: A novel bufalin derivative exhibited stronger apoptosis-inducing effect than bufalin in A549 lung cancer cells and lower acute toxicity in mice. PLoS One 11: e0159789, 2016

32. Wu SH, Bau DT, Hsiao YT, Lu KW, Hsia TC, Lien JC, Ko YC, Hsu WH, Yang ST, Huang YP and Chung JG: Bufalin induces apoptosis in vitro and has antitumor activity against human lung cancer xenografts in vivo. Environ Toxicol 32: 1305-1317, 2017.
33. Xie XB, Yin JQ, Wen LL, Gao ZH, Zou CY, Wang J, Huang G, Tang QL, Colombo C, He WL, et al: Critical role of heat shock protein 27 in bufalin-induced apoptosis in human osteosarcomas: A proteomic-based research. PLoS One 7: e47375, 2012 .

c) (†) $\Theta$ This work is licensed under a Creative Commons (c) ${ }_{\mathrm{EY} \text { NC ND }}$ Attribution-NonCommercial-NoDerivatives 4.0 International (CC BY-NC-ND 4.0) License. 